\title{
ANÁLISE DAS TENDÊNCIAS ESPAÇO-TEMPORAIS DAS PRECIPITAÇÕES ANUAIS PARA O ESTADO DO PARANÁ - BRASIL
}

\author{
ELY, Deise Fabiana - deise.ely@gmail.com \\ Universidade Estadual de Londrina/UEL \\ DUBREUIL, Vincent - vincent.dubreuil@uhb.fr \\ Université Rennes 2
}

\begin{abstract}
RESUMO: O presente trabalho sintetiza as análises dos resultados da aplicação de uma modelagem geoestatística dos totais pluviométricos anuais, oriundos do Instituto Agronômico do Paraná (IAPAR), do Instituto Nacional de Meteorologia (INMET) e da Agência Nacional de Águas (ANA), com o intuito de identificar as tendências espaçotemporais das chuvas no estado do Paraná obtidas por meio dos testes da Correlação Linear e de Mann-Kendall; calculadas ao nível dos pixels dos mapas, com uma resolução espacial de um quilômetro. Foi verificado que as tendências estatisticamente significativas observadas no estado são aquelas que indicam aumento nos totais pluviométricos anuais. Enquanto que as tendências à redução têm uma abrangência espacial maior, porém não significativas. As áreas que apresentaram tendências de aumento nos testes da Correlação Linear e de Mann-Kendall são as mesorregiões: Metropolitana (mais especificamente Curitiba e o norte do litoral paranaense), CentroSul, Oeste (Catanduvas), Centro-Ocidental (Altamira do Paraná), Norte Central e Norte Pioneiro. E aquelas onde os totais anuais tendem a diminuir são: Centro-Oriental, Norte Central (Maringá e Califórnia), Noroeste, Sudeste e o extremo oeste da mesorregião Oeste
\end{abstract}

Palavras- chaves: modelagem geoestatística; tendências; precipitações anuais; Paraná.

ANALYSIS OF SPATIOTEMPORAL TRENDS OF ANNUAL PRECIPITATION FOR PARANÁ STATE - BRAZIL

ABSTRACT: This paper summarizes the analysis of the results of applying the geostatistical modeling of annual rainfall data, derived from the Agronomic Institute of Paraná (IAPAR), the National Institute of Meteorology (INMET) and National Water Agency (ANA), aiming to identify the spatiotemporal trends of rainfall in the state of Paraná by tests of linear correlation and Mann-Kendall. Spatiotemporal trends were calculated in terms of pixels of the maps with a spatial resolution of one kilometer. The most significant trends in the state are those indicating increased annual precipitation. The reducing trends in annual precipitation cover a larger area of the state, but the rates have no strong significance. The mesoregions showed increasing trends in tests of linear correlation and Mann-Kendall: Metropolitan region (specifically Curitiba and the northern coast of Paraná), Central South, West (Catanduvas), Central West (Altamira do Paraná), North Central and North. Annual rainfall tends to decrease in East-Central, North Central (Maringa and California), Northwest, Southeast and the west end of West mesoregion.

Keywords: geostatistical modeling; trends; annual rainfall; Paraná.

\section{ANALYSE SPATIOTEMPORELLE DES TENDANCES DES PRÉCIPITATIONS ANNUELLES DANS L'ETAT DU PARANÁ, BRÉSIL}

RÉSUMÉ: Cet article présente une analyse des résultats de la modélisation des données de précipitations annuelles, provenant de I'Institut Agronomique du Paraná (IAPAR), l'Institut national de météorologie (INMET) et l'Agence nationale de l'eau (ANA), afin d'identifier les tendances spatio-temporelles des précipitations dans I'Etat du Paraná. Ce travail a pu être réalisé à l'échelle de pixels avec une résolution spatiale du un kilomètre en raison de la forte densité de stations avec des données pluviométriques disponibles pour l'Etat. Il a été constaté que les tendances les plus significatives de l'Etat sont celles qui indiquent une augmentation des précipitations annuelles, mais seulement pour 
certaines régions. En revanche, la tendance à la réduction des précipitations annuelles couvre une plus grande surface de l'État, mais avec des taux plus modestes et non significatifs. Les régions qui ont montré une tendance croissante dans les tests de corrélation linéaire et Mann-Kendall sont : la région Métropolitaine (Curitiba et la côte nord du Paraná), le Centre-Sud et l'Ouest (Catanduvas), le Centre-Ouest (Altamira do Paraná ), le Centre-Nord et la région pionnière nord. Les méso-régions où les précipitations annuelles ont tendance à diminuer sont: le Centre-Est, le Centre-Nord (Maringa et Californie), le Nord-Ouest, le Sud-Est et l'extrémité ouest de la méso-région Ouest.

Mots Clés: Modéliation géostatistique, tendances, pluies annuelles, Paraná.

\section{ANÁLISIS DE LAS TENDENCIAS ESPACIO-TEMPORALES DE LAS PRECIPITACIONES ANUALES PARA EL ESTADO DE PARANÁ - BRASIL}

RESUMEN: El presente trabajo sintetiza los análisis de los resultados de la aplicación del modelaje de datos pluviométricos anuales, oriundos del Instituto Agronômico do Paraná (IAPAR), del Instituto Nacional de Meteorologia (INMET) y de la Agência Nacional de Águas (ANA), con la intención de identificar las tendencias espacio-temporales de las lluvias en el estado de Paraná; calculadas al nivel de los píxeles de los mapas, con una resolución espacial de un quilómetro posibilitado por la alta densidad de las estaciones con datos de precipitaciones disponibles para el estado. Fue verificado que las tendencias más expresivas observadas en el estado son aquellas que indican aumento de las precipitaciones anuales, pero para áreas más específicas. Mientras que las tendencias de reducción en las precipitaciones anuales abarcan una área mayor del estado, sin embargo con indicadores más modestos. Las áreas que presentan tendencias de aumento en los exámenes de la Correlación Linear y de Mann-Kendall son las mesorregiones: Metropolitana (más específicamente Curitiba y el norte del litoral paranaense), Centro-Sur, Oeste (Catanduvas), Centro-Occidental (Altamira do Paraná), Norte Central y Norte Pioneiro. Y las mesorregiones en que las precipitaciones anuales tienden a disminuir son: Centro-Oriental, Norte Central (Maringá y Califórnia), Noroeste, Sudeste y el extremo oeste de la mesorregión Oeste.

Palabras Clave: modelaje geoestadística; tendencias, precipitaciones anuales; Paraná.

\section{INTRODUÇÃO}

Muitas das várias atividades econômicas desenvolvidas pela sociedade são influenciadas pelas condições climáticas em diversos graus. Especialmente nas atividades agrícolas o clima é tido como um condicionante, pois o desenvolvimento das culturas é compatibilizado com o tipo de oferta climática, que é variável no tempo e no espaço.

Dubreuil et al (2012) esclarecem que a variabilidade de um dado parâmetro climático pode se dar mais ou menos em torno de um valor de referência central (da normal climatológica, por exemplo), ou seja, por meio de sua análise é possível observar os anos mais ou menos quentes ou mais úmidos e secos. E destacam que o desenvolvimento técnico das sociedades permitiu sua adaptação a estes referenciais climáticos. No tocante à variação climática os autores se referem a uma alteração progressiva e duradoura no clima, tornando-se década após década mais frio ou mais quente. Esta não é a observação de um ou dois anos, mesmo se ela for consecutiva e, para afirmar a ocorrência desse tipo de tendência são necessárias observações durante um longo período de tempo, da ordem de meio século.

A variabilidade constitui um aspecto natural do clima e influencia diretamente as atividades socioeconômicas; justificando seu estudo pela Climatologia e pela Geografia, bem como das tendências climáticas. 
De acordo com Nascimento Júnior; Silvestre; Dubreuil e Sant'Anna Neto (2013) a análise e a descrição dos fenômenos meteorológico-climáticos é efetuada por meio de dados numéricos, que prescinde da utilização de técnicas estatísticas (distribuição de frequência, medidas de tendência central, de dispersão, de variabilidade, correlações, dentre outras), representações gráficas e a construção de modelos que representem sua repercussão no território. Os autores ainda destacam que "O intuito da utilização dessas técnicas é a definição de (des)continuidades, (a)ritmias, variações de máximas e mínimas, que podem ocorrer de forma sequencial ou não, e/ou com ausência/presença de padrão específico, inerente à ideia e ao conceito de variabilidade climática" (2013, p. 2).

Diante do exposto, o presente trabalho apresenta as análises dos resultados da aplicação de uma modelagem geoestatística dos totais pluviométricos anuais (1977 a 2014) oriundos do Instituto Agronômico do Paraná (IAPAR), do Instituto Nacional de Meteorologia (INMET) e da Agência Nacional de Águas (ANA), bem como as tendências espaço-temporais das chuvas no estado do Paraná; calculadas ao nível dos pixels dos mapas produzidos com uma resolução espacial de um quilômetro propiciada pela alta densidade de postos e estações com dados pluviométricos diários.

\section{VARIABILIDADE CLIMÁTICA DO ESTADO DO PARANÁ}

A variabilidade espaço-temporal do clima tem sido estudada em variadas escalas e, especificamente para a América do Sul, foram verificadas variações no ciclo hidrológico e na temperatura média (SILVA; GUETTER, 2003). Barros (2010) ressalta que a América do Sul meridional tem mostrado tendências positivas na precipitação durante o último século, além do aumento na precipitação média anual, episódios de chuvas intensas estão se tornando mais frequentes.

O Paraná está localizado na confluência dos principais sistemas atmosféricos da América do Sul e possui mais de um regime pluviométrico. Mendonça (1994) destaca que o Paraná apresenta uma distribuição pluvial anual com chuvas concentradas de setembro a abril com tendência ao regime tropical nas porções norte e noroeste e subtropical nas demais porções.

Dubreuil; Fante; Planchon e Sant'Anna Neto (2017), subsidiados na análise das temperaturas e precipitações médias anuais e de acordo com a classificação climática de Köppen, caracterizam o tipo climático Cfa como predominante no Paraná, ou seja, um clima subtropical com temperatura média no mês mais frio inferior a $18^{\circ} \mathrm{C}$ e temperatura média no mês mais quente acima de $22^{\circ} \mathrm{C}$, com verões quentes, geadas pouco frequentes e com tendência de concentração das chuvas no verão, mas sem estação seca definida.

Mendonça (2006) destaca que, em termos gerais, estima-se que nos últimos quarenta anos a temperatura média na região sul do Brasil elevou cerca de $1,3^{\circ} \mathrm{C}$, o que teria sido acompanhado por uma elevação dos totais pluviométricos médios anuais. Mas essa intensificação do aquecimento regional é espacial e termicamente diferenciada e esclarece que tal intensificação se processou de maneira mais explícita na porção norte da região sul do Brasil, ou seja, no estado do Paraná; onde o reforço das condições de tropicalidade tem se 
evidenciado por meio de invernos menos frios e chuvas mais concentradas nas últimas décadas.

Assim como as tendências térmicas, as análises das tendências pluviométricas para a região sul do Brasil a partir de uma série de dados de 38 localidades (oito situadas no Paraná) permitiram Mendonça (2014) indicar que as alterações são mais evidentes na porção norte regional, ou seja, no estado do Paraná.

\begin{abstract}
Observando-se, particularmente, o contexto pluviométrico do estado do Paraná constata-se que a tendência de elevação dos totais médios anuais é mais representativa na porção centro-sul (da ordem de 100 a $300 \mathrm{~mm}$ ), especialmente ao longo da grande bacia do rio Iguaçu...
\end{abstract}

$\mathrm{Na}$ porção centro-norte desse estado foi registrada tendência à redução pluviométrica, de cerca de até $200 \mathrm{~mm}$, opostamente à porção centrosul. (MENDONÇA, 2014, p. 34)

Minuzzi e Caramori (2011, p. 596) identificaram que, "[...] desde meados do século XX o Estado do Paraná apresenta uma tendência de aumento na quantidade de chuvas, principalmente, durante o verão e a primavera", com incrementos de 17 a $37 \mathrm{~mm} /$ década. Destacam que no litoral do estado, especialmente em Antonina e Morretes, desde meados da década de 1940, a quantidade de chuva no verão e na primavera aumentou em torno de 177 e $226 \mathrm{~mm}$, respectivamente. Ainda enfatizam que as estações com maior número de tendências significativas, na primavera e na escala anual, estão concentradas no leste do estado, ou seja, em áreas mais próximas ao oceano Atlântico e com algumas eventuais áreas a sudoeste.

Ely e Dubreuil (2014) analisaram a variabilidade interanual das temperaturas e precipitações na vertente sul da bacia hidrográfica do Rio Paranapanema com o objetivo de identificar uma possível variação climática. Os dados diários de temperatura e precipitação foram organizados em gráficos que apresentam a variabilidade interanual para o período de 1979 a 2010 e foram submetidos aos testes estatísticos da regressão linear, Mann-Kendall e Pettitt. Foi identificado que a variabilidade espaço-temporal das temperaturas é regulada pela latitude, com a ocorrência de dois regimes térmicos distintos na área (clima tropical no centro-norte e subtropical no sul). O teste estatístico da regressão linear indicou tendência ao aumento das temperaturas de forma geral. Enquanto que os testes de Mann-Kendall e Pettitt revelaram a não ocorrência de tendência nas precipitações anuais na vertente sul da bacia do Rio Paranapanema.

Carmello; Dubreuil e Sant'Anna Neto (2015) também estudaram a distribuição espacial dos totais anuais das chuvas na vertente sul da bacia hidrográfica do Rio Paranapanema por meio de uma análise estatística dos anos secos (1999-2000) e chuvosos (2009-2010). Foram utilizados dados pluviométricos mensais de 89 postos e estações que foram interpolados por meio do método da krigagem e submetidos à análise geoestatística. Os resultados para a área de estudo indicaram que o ano agrícola 2009-2010 registrou os maiores valores de precipitação, atingindo um volume total de $1.776 \mathrm{~mm}$ na parte oriental e de $1.047 \mathrm{~mm}$ no sudeste da bacia. O menor total pluviométrico foi verificado para o ano agrícola 1999-2000 (477mm), sendo que 
o maior observado $(1.153 \mathrm{~mm})$ neste ano ocorreu no leste e no interior do norte da bacia.

Nascimento Júnior; Silvestre; Dubreuil e Sant'Anna Neto (2013) buscaram verificar a existência ou não de rupturas e tendências estatísticas nos valores de precipitação para o estado do Paraná. Para tanto, utilizaram dados de 63 postos pluviométricos que foram organizados em totais mensais (CHA) e em número de dias com chuva (NDC) para as escalas trimestrais, sazonais e anuais; submetidos aos testes de Pettitt, de Mann-Kendall e ao modelo de Regressão Linear. Os resultados demonstraram que a década de 1990 constitui o principal período de transição dos valores de CHA e NDC no estado, indicando aumento do primeiro e diminuição do segundo. Para o NDC foi observado um aumento em quase todas as escalas (com exceção do verão), mas os totais de chuva somente apresentaram um incremento na estação chuvosa e no verão. A redução de CHA e NDC foi observada, principalmente, no outono e na escala anual. As análises realizadas indicam uma tendência à concentração das chuvas no estado do Paraná nos últimos 36 anos.

Vale ressaltar que a chuva é um fenômeno meteorológico que apresenta ampla variabilidade temporal e espacial e requer uma análise detalhada de quais controles geográficos regem esse mosaico climático.

Os estudos de HAYLOCK et al (2006) também têm revelado um padrão nas tendências da ocorrência de eventos extremos e na quantidade anual das chuvas para a América do Sul nas últimas décadas. O último relatório do IPCC (2014) enfatiza que, possivelmente sejam mais numerosas as regiões continentais onde o número de episódios de precipitações extremas mais aumentou que diminuiu; que os eventos extremos de precipitação se tornem mais frequentes e intensos na maior parte das áreas localizadas nas latitudes médias continentais e nos trópicos úmidos, mas que em muitas áreas das latitudes médias e nas regiões subtropicais secas a precipitação média irá diminuir.

O Paraná tem sua economia atrelada, principalmente, à produção agrícola que depende de um conhecimento mais aprofundado de sua variabilidade climática; sobretudo da pluviométrica. O estado também concentra um expressivo número de usinas hidrelétricas que necessitam de análises climáticas para o controle dos níveis e vazão dos reservatórios. Além disso, Mendonça (2006 e 2014) identificou que o clima nesta escala vem apresentando os efeitos do aquecimento global, ou seja, intensificação do aquecimento com incremento nas taxas de umidade e, consequentemente, da pluviosidade; configurando-se um desafio estudar e ampliar o mapeamento de possíveis mudanças no total anual das chuvas sobre este recorte espacial.

Considerando a recente evidência de tendências ao aumento dos episódios de precipitações extremas que desencadeiam 0 aumento do escoamento em bacias hidrográficas e do risco de inundações em escalas regionais, o citado relatório (IPCC, 2014) menciona a necessidade de estudos que analisem a variabilidade climática regional, pois as alterações na precipitação não serão uniformes. Assim, o presente artigo tem o objetivo de analisar a distribuição espaço-temporal dos totais pluviométricos anuais no estado do Paraná e de suas tendências a partir de uma modelagem geoestatística das séries históricas do IAPAR, do INMET e da ANA que, em 
conjunto, propiciam uma maior densidade espacial de dados; requisito para a aplicação da referida modelagem.

\section{METODOLOGIA}

O desenvolvimento do presente trabalho partiu da sistematização dos dados pluviométricos diários oriundos das redes de coleta do IAPAR, do INMET e da ANA em planilhas anuais. Foram coletados e sistematizados os dados de 605 estações e postos pluviométricos distribuídos no estado, mas devido às falhas nas séries e tendo como critério de manter na análise as estações e postos que tivessem até $10 \%$ de dados faltantes para que se pudesse ter uma maior abrangência espacial dos mesmos, foram manipulados os dados pluviométricos de 330 postos e estações (figura 1), sendo 323 localizadas no Paraná e 7 nos limites dos estados de São Paulo, Santa Catarina e Mato Grosso do Sul, para o período de 1977 a 2014.

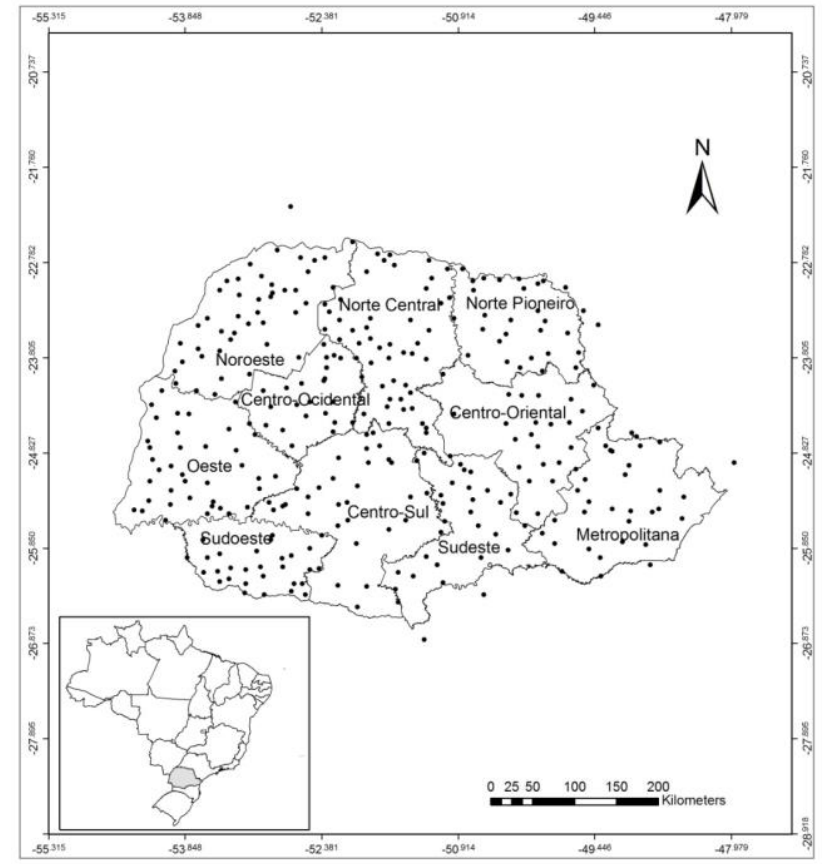

Figura 1 - Localização dos postos / estações. (Elaborado pela autora.)

Por meio da planilha eletrônica do Windows Office Excel 2007 foi gerado um banco de dados com os totais pluviométricos anuais. Posteriormente, essa planilha foi exportada para o software de Sistema de Informação Geográfica (SIG) IDRISIC (1987-2012 Clark University, versão Selva 17.00) e com o objetivo de representar a variabilidade espacial das chuvas anuais sobre o estado, considerando que os dados trabalhados representam valores pluviométricos registrados em um determinado ponto no espaço, foi produzida a espacialização das chuvas sobre a área estudada. Esta operação cartográfica foi executada por meio da interpolação dos valores registrados em cada posto e estação. De acordo com Carmello; Dubreuil e Sant'Anna Neto (2015, p. 441442), esse processo permite estimar o valor da variável estudada num ponto não amostrado. O método de interpolação utilizado foi o índice de distância ao ponto vizinho mais próximo, "[...] definido pela razão entre a distância média 
calculada de cada ponto até o vizinho mais próximo (LO) e a distância média esperada entre todos os pontos do mapa (Le)" (FERREIRA, 2014, p. 123).

Numa etapa seguinte, estes mapas foram transformados em formato raster, ou seja, foi gerada uma imagem constituída por uma malha fina de células de grade (pixels) que incorporam um valor numérico; o valor de precipitação oriundo da interpolação realizada. Cada pixel das imagens geradas tem uma resolução espacial de $1 \mathrm{~km} \mathrm{X} 1 \mathrm{~km}$. Outra etapa constituiu a submissão dessas imagens a um filtro digital. No IDRISIC) podem ser aplicados filtros de $3 \times 3,5 \times 5,7 \times 7$ ou outras opções definidas pelo usuário. Os filtros digitais produzem uma operação matemática (que pode ser a média, Gaussiana, mediana, desvio padrão, dentre outras ou, até mesmo, definidas pelo operador do SIG) entre o valor da célula original e de seus vizinhos mais próximos radialmente com o objetivo de extrapolar o valor da célula para uma área maior.

Na modelagem realizada neste estudo foi empregado o filtro $5 \times 5$, com a opção do cálculo da média entre a célula original e as vizinhas. Esta operação foi possível em virtude da alta densidade de postos e estações com dados pluviométricos disponíveis para o Paraná.

Após esse processamento, as imagens foram agrupadas por meio do módulo "Earth Trends Modeler (ETM)", ferramenta que permite análises da dinâmica e das tendências características de fenômenos que são evidenciados por uma sequência temporal de imagens e instrumental que possibilita a análise de tendências de longo prazo.

São sete as opções de saídas do módulo ETM do IDRISIC. No presente estudo são analisados os resultados de cinco testes que apresentaram desempenhos mais significativos. O conjunto de imagens geradas foi submetido ao teste da regressão linear que, conforme Back (2001), pode ser utilizado para indicar alterações climáticas por meio do teste de significância do coeficiente angular, que consiste em determinar o intervalo de confiança do coeficiente "a".

O procedimento do cálculo da correlação linear identifica o coeficiente de determinação (R2) a partir de uma regressão linear entre os valores de cada pixel ao longo do tempo para uma determinada série linear. No IDRISIC este cálculo considera a correlação linear de Pearson entre os valores de cada pixel ao longo da série histórica espacialmente distribuída.

O teste de Mann-Kendall é um teste não paramétrico utilizado para identificar mudanças climáticas em séries temporais de dados que, na hipótese da estabilidade de uma série temporal, os valores devem ser independentes e a distribuição de probabilidades deve permanecer sempre a mesma (série aleatória simples). Na aplicação do teste estatístico de Mann-Kendall a série de dados é a variável dependente e o tempo é a variável independente. Quando a variável independente é algo diferente do tempo, a estatística é conhecida como Tau de Kendall (que pode ser calculada no módulo ETM do IDRISI@). Nesse caso, é possível verificar se as duas variáveis estão ambas aumentando ou diminuindo (conhecida como uma concordância) ou se uma aumenta enquanto a outra diminui (uma discordância) entre todas as combinações de pares de observações. Tau é, então, a frequência relativa de concordâncias menos a frequência relativa de discordâncias. (HAYLOCK et al, 2006)

No referido módulo do IDRISI@ foi aplicado o teste de Mann-Kendall para detectar tendências monotônicas, que fornece um indicador de tendência não 
linear que mede o grau em que a tendência é consistentemente crescente ou decrescente e varia no intervalo de -1 a 1 . Um valor 1 indica uma tendência que aumenta continuamente e nunca diminui. O inverso é verdadeiro, quando se tem um valor de -1 . Um valor 0 indica que não há tendência consistente (HAYLOCK et al, 2006). Todas as combinações de pares de valores ao longo do tempo são avaliadas para cada pixel e uma contagem é feita para verificar se o número tende a aumentar ou a diminuir com o tempo. (SALVIANO; GROPPO; PELEGRINO, 2016)

O teste de Mann-Kendall usado para detectar tendências monotônicas (de aumento ou diminuição) é amplamente utilizado para detectar tendências em séries temporais porque é simples, robusto, se adapta aos dados faltantes e os dados não precisam estar em conformidade com qualquer distribuição estatística. Em havendo chances de inconstâncias na série de dados, o teste não paramétrico de Mann-Kendall é útil, pois sua estatística está baseada nos sinais (+ ou -), ao invés de valores de variável randômica e, por conseguinte, as tendências determinadas são menos afetadas pelas inconsistências. (CHAKRABORTY; PANDEY; CHAUBE; MISHRA, 2013)

A opção do teste de significância de Mann-Kendall produz imagens com valores $Z$ que possibilitam a medição tanto da significância quanto a direção da tendência simultaneamente. Quando os valores excedem o limite crítico determinado pelo nível de significância escolhido, que no presente estudo é de $10 \%$, aceita-se a existência significativa da tendência. Este teste é comumente usado como um teste de tendência assim como o estimador Theil-Sen $(\beta)$.

A opção Theil-Sen, além de identificar se existe uma tendência, permite que a magnitude desta possa ser estabelecida por um estimador de declividade mediana $\beta$; um estimador robusto da magnitude da tendência. Em outras palavras, a declividade mediana $\beta$ é a mediana sobre todos os pares de combinações possíveis para a totalidade da série de dados. Um valor positivo de $\beta$ indica uma tendência ascendente (aumento dos valores com o tempo), enquanto que um valor negativo de $\beta$ indica uma tendência à redução. (CHAKRABORTY; PANDEY; CHAUBE; MISHRA, 2013)

Os resultados da aplicação da declividade mediana para uma série longa são, frequentemente, idênticos à saída da tendência linear (OLS). No entanto, para uma série curta ou muito ruidosa, o resultado pode ser completamente diferente e mais exato.

O significado da declividade mediana pode ser estimado usando uma medida de pixel baseada no teste chamado de significância de Mann-Kendall (MK) ou utilizando o teste de significância contextual de Mann-Kendall (CMK) que consiste numa regionalização desse teste e que pode ser aplicado em séries históricas de imagens para cada um de seus pixels. As duas opções fornecem um $Z$ normalizado e uma probabilidade correspondente às imagens $(P)$ como um resultado.

A opção de tendência linear (OLS) mapeia o coeficiente de declividade dos ordinários mínimos quadrados de regressão entre os valores de cada pixel ao longo do tempo para uma série perfeitamente linear. O resultado é uma expressão da taxa de variação por intervalo de tempo. Assim, se seus dados são anuais, ela expressa a taxa de ganho anual. 
Os métodos estatísticos descritos para a verificação de tendências foram utilizados, por exemplo, por Debortoli; Dubreuil; Henke e Rodrigues Filho (2012) para identificar tendências e rupturas em séries pluviométricas da Amazônia brasileira meridional. Os autores identificaram que, das 207 estações submetidas ao teste de Mann-Kendall, 85 estações apresentaram tendências negativas e 25 positivas; propiciando verificar que as mudanças mais significativas ocorrem nos meses das estações de transição (outono e primavera). A regressão linear permitiu identificar tendências negativas para as precipitações em 136 estações e em 76, tendências positivas. Os autores concluíram que a redução na precipitação parece estar concentrada no início e no final da estação chuvosa, afetando modestamente as chuvas de maneira geral.

Tozato; Dubreuil e De Mello-Théry (2013) aplicaram os teste de MannKendall, Pettitt e regressão linear às séries anuais dos dados de chuva, temperatura e cotas fluviométricas de estações localizadas na sub-bacia do Alto Paraguai para o período de 1971 a 2011. E constataram redução nas chuvas anuais na porção que se estende da Amazônia ao centro do Pantanal e aumento das temperaturas máximas e mínimas nas estações de Cáceres e Cuiabá, ambas localizadas no estado de Mato Grosso. Foi identificada uma diminuição no nível da água dos rios São Lourenço, Cuiabá e Paraguai; indicando que a variabilidade climática é responsável apenas por parte da variação das cotas fluviométricas, pois alguns rios apresentaram aumento nesses valores.

Assim como Nascimento Júnior, Silvestre, Dubreuil e Sant'Anna Neto (2013); Ely e Dubreuil (2014); Carmello, Dubreuil e Sant'Anna Neto (2015), dentre outros, consideram a variabilidade a partir dos dados de cada posto ou estação para a identificação de tendências para diferentes regiões do Brasil e do mundo.

Vale destacar que no presente estudo foram produzidas análises a partir do conjunto de dados pluviométricos da série histórica de 1977 a 2014 objetivando verificar a distribuição espaço-temporal das chuvas anuais no estado do Paraná e de suas tendências espaciais por meio da modelagem descrita, ou seja, as tendências são calculadas ao nível dos pixels dos mapas e não a partir das séries pontuais; como é feito na maioria dos trabalhos.

\section{DISCUSSÃO DOS RESULTADOS}

A partir dos dados pluviométricos diários das redes do IAPAR, do INMET e da ANA foi produzida a série temporal das precipitações anuais para o período de 1977 a 2014. Por meio desta série foram gerados os mapas dos totais pluviométricos anuais que, posteriormente, foram agrupados e submetidos aos testes estatísticos disponíveis no módulo "Earth Trends Modeler (ETM)" do IDRISIC para a identificação das tendências espaço temporais das chuvas anuais no estado do Paraná.

De acordo com o resultado do teste da Correlação Linear (figura 2) e considerando o valor mínimo de $r$ para uma significância de $10 \%$, conforme Rogerson (2012), pode-se confirmar a afirmação do IPCC (2014) de que as variações das precipitações não são uniformes no cenário de um mundo que aquece. Verifica-se que os totais anuais das precipitações no estado do Paraná apresentam tendências negativas e positivas. No entanto, as tendências 
negativas não são significativas pois estão fora do valor crítico de referência; que é de 0,264. A mesorregião metropolitana registra tendências significativas para o aumento das chuvas anuais (especialmente próximo à capital e norte do litoral, com índices superiores a 0,40$)$. O sul da mesorregião Centro-Oriental $(0,38)$, a mesorregião Centro-Sul $(0,39)$ e as mesorregiões Centro-Ocidental $(0,40)$ e Norte Central $(0,38)$ também apresentam significativas tendências positivas para as chuvas anuais.



Figura 2 - Tendências das Precipitações anuais no estado do Paraná (Correlação Linear) no período de 1977 a 2014. Fonte: IAPAR, INMET e ANA (2015) - Elaborado pela autora.

A figura 3 apresenta o resultado da modelagem do teste de Mann-Kendall tendência monotônica. Haylock et al (2006) esclarecem que o Tau de Kendall é expresso em valores que oscilam entre -1 e 1 e que tem a vantagem de permitir a comparação de índices entre estações com quantidades de chuvas muito diferentes. Moreira; Naghettini (2016) complementam que, apesar da eficiência do teste de Mann-Kendall, ele não fornece a magnitude das tendências detectadas. Assim, no presente estudo foi constatado que a aplicação deste proporciona uma caracterização mais precisa das áreas que não apresentam tendências, bem como daquelas com tendências positivas ou negativas. As mesorregiões Centro-Oriental, Sudeste, Sudoeste, Oeste, Nordeste, CentroOcidental e Norte-Central se destacam pelas tendências negativas, mas que não são significativas. Enquanto que as tendências positivas são reforçadas para o Norte da mesorregião Metropolitana, para o Sul da mesorregião CentroOcidental e para o leste da mesorregião Centro-Oriental (com índices superiores a 0,30$)$, extremo norte da mesorregião Norte-Central $(0,28)$ e na mesorregião Centro-Sul $(0,26)$. 


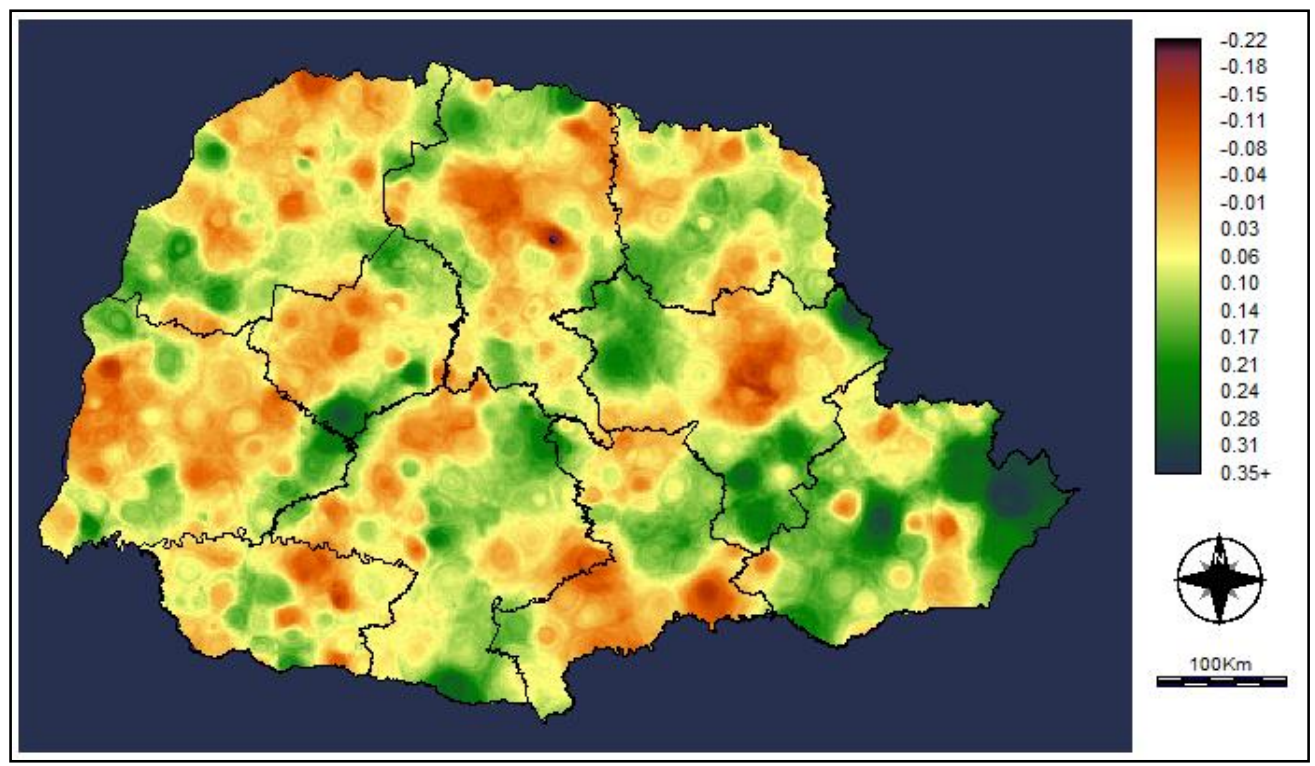

Figura 3 - Resultado do teste de tendência monotônica de Mann-Kendall para as precipitações anuais no estado do Paraná no período de 1977 a 2014. Fonte: IAPAR, INMET e ANA (2015) - Elaborado pela autora.

$\mathrm{Na}$ figura 4 são destacadas algumas localidades que apresentaram tendências nos totais anuais das precipitações (positivas ou negativas conforme indica a inclinação da reta de tendência nos gráficos dos totais pluviométricos anuais). Os municípios de Curitiba e Morretes (na mesorregião Metropolitana) apresentam índices positivos mais significativos (superiores a 0,33). O município de Altamira do Paraná, na mesorregião Centro-Ocidental, se destaca por um índice positivo de 0,28; assim como o município de Alvorada do Sul (na mesorregião Norte-Central, com índice de 0,28 ). Enquanto que os municípios de Maringá (mesorregião Norte-Central), Iguatu (mesorregião Oeste), São Jorge d'Oeste (mesorregião Sudoeste) e São Mateus do Sul (mesorregião Sudeste) se destacam pelos valores negativos; mas que não são significativos $(-0,04$ a $0,18)$. 


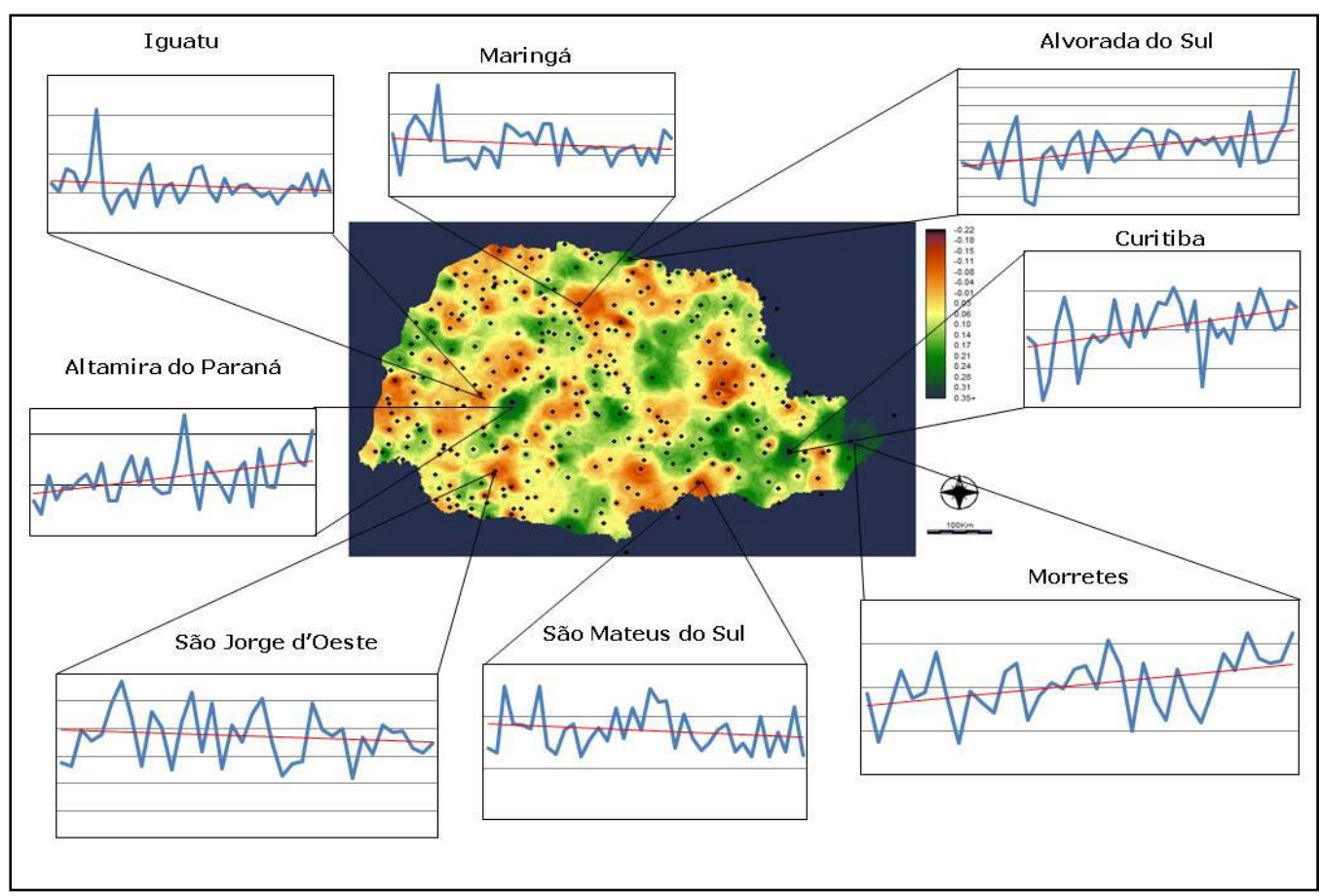

Figura 4 - Detalhamento dos resultados do teste de tendência monotônica de MannKendall para algumas localidades do estado do Paraná.Fonte: IAPAR, INMET e ANA (2015) - Elaborado pela autora.

A partir dos resultados apresentados, ressalta-se que as chuvas anuais no estado do Paraná apresentam uma variabilidade característica deste fenômeno meteorológico, mas com áreas em que os valores são mais significativos para o aumento do que para a redução.

Com o teste de significância de Mann-Kendall (figura 5) pode-se constatar com maior clareza as áreas com tendências negativas (as mesmas já destacadas para o teste de Mann-Kendall tendência monotônica) e uma ampliação daquelas com tendências positivas. Vale destacar que as áreas com índices entre -1,65 e 1,65, para uma significância de 10\% conforme Ferreira; Penereiro e Fontolan (2015), são as que apresentaram tendências significativas tanto para a redução quanto para o aumento dos totais pluviométricos anuais. 


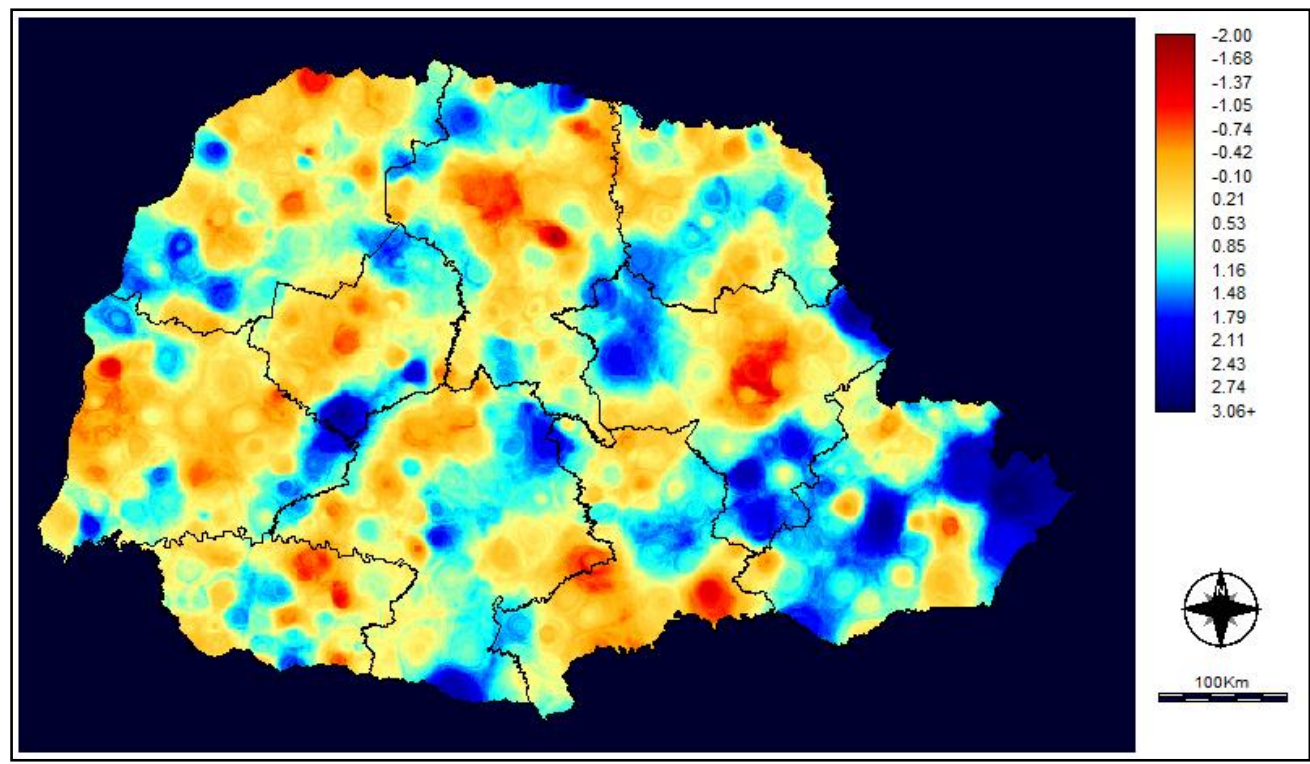

Figura 5 - Resultado do teste de significância de Mann-Kendall para as precipitações anuais no estado do Paraná no período de 1977 a 2014.Fonte: IAPAR, INMET e ANA (2015) - Elaborado pela autora.

Como mencionado na metodologia, os resultados da espacialização dos testes da declividade mediana (Theil-Sen) e da tendência linear (OLS) são muito próximos para uma longa série de dados (vide as espacializações apresentadas na figura 6 ).
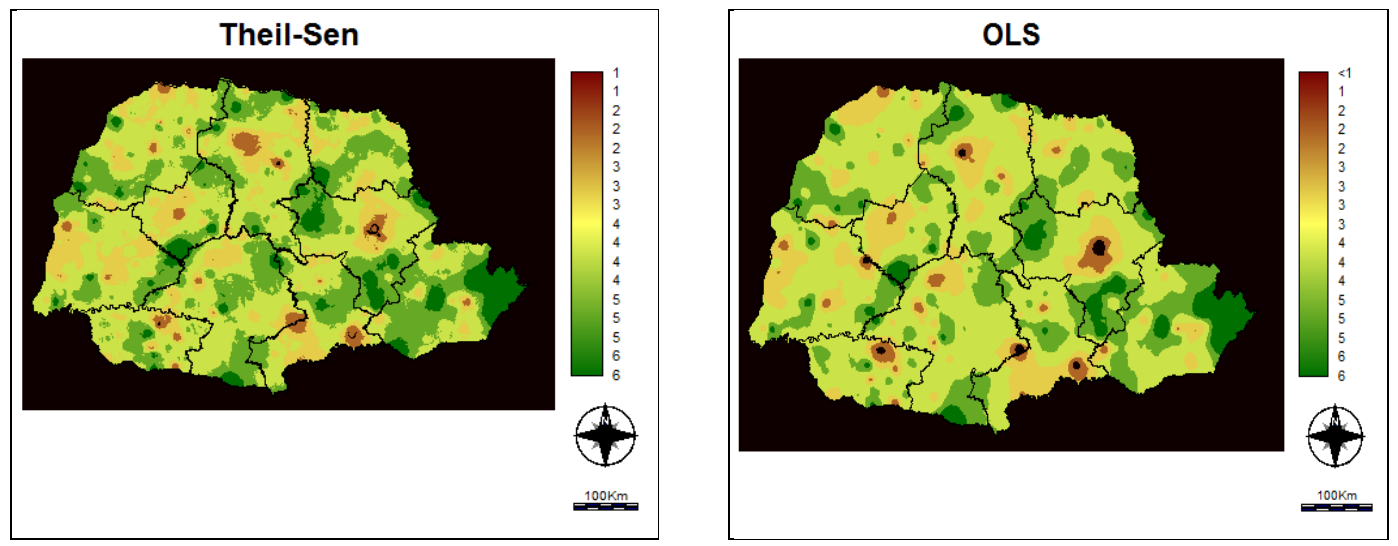
Informações adicionais às Legendas
Classe 1: de $-10,00$ a $-6,50$
Classe 4: de $-0,05$ a 4,00
Classe 2: de $-6,50$ a $-3,50$
Classe 5: de 4,00 a 8,00
Classe 3: de $-3,50$ a $-0,05$
Classe 6: de 8,00 a 18,00

Figura 6 - Resultado dos testes de declividade mediana (Theil-Sen) e tendência Linear (OLS) para as precipitações anuais no estado do Paraná no período de 1977 a 2014. Fonte: IAPAR, INMET e ANA (2015) - Elaborado pela autora. 
Vale frisar que a tendência linear (OLS) expressa a taxa de ganho ou perda anual para as precipitações no estado, ou seja, Curitiba, por exemplo, apresenta um ganho anual de 17,26mm; Paranaguá de 16,82; Altamira do Paraná de 17,14; Alvorada do Sul, no extremo Norte do estado, apresenta um acréscimo de $14,16 \mathrm{~mm}$ em suas precipitações anuais enquanto que Palmas, no extremo Sul, um aumento de $12,87 \mathrm{~mm}$.



Figura 7 - Detalhamento dos resultados do teste de tendência linear (OLS) para algumas localidades do estado do Paraná.Fonte: IAPAR, INMET e ANA (2015) - Elaborado pela autora.

A partir dos resultados dispostos nas figuras 6 e 7 constata-se que a maior parte do estado apresenta reduções nas precipitações anuais, mas com valores modestos que variam de $-1,50$ a $-10,22 \mathrm{~mm}$ ao longo do período analisado, sobressaindo algumas áreas da mesorregião Centro-Oriental como, por exemplo, o município de Reserva com valores de $-9,98 \mathrm{~mm}$; São Mateus do Sul $(-7,16 \mathrm{~mm})$ na mesorregião Sudeste; São Jorge do D'Oeste $(-8,06 \mathrm{~mm})$ na mesorregião Sudoeste e Maringá $(-7,92 \mathrm{~mm})$ no Norte Central.

\section{CONSIDERAÇÕES FINAIS}

A metodologia desenvolvida e aplicada à análise espaço temporal (1977 a 2014) dos totais pluviométricos anuais no estado do Paraná mostrou-se relevante para o alcance dos objetivos propostos, pois permitiu demonstrar que as áreas que registram redução são mais abrangentes, mas os índices estatísticos obtidos não são significativos. Enquanto que as áreas que registram 
aumento são mais restritas, porém com índices significativos. Bem como propiciou um detalhamento das tendências identificadas para as diferentes mesorregiões do estado.

As áreas que apresentaram tendências de aumento nos testes da Correlação Linear e de Mann-Kendall são as mesorregiões: Metropolitana (mais especificamente Curitiba e o norte do litoral paranaense), Centro-Ocidental (Altamira do Paraná) e Norte Central; algumas coincidem com aquelas identificadas por Mendonça (2014) e Minuzzi e Caramori (2013). E com a aplicação do teste da tendência Linear (OLS) foram obtidos incrementos superiores a $400 \mathrm{~mm}$ nos totais pluviométricos anuais em algumas áreas do Paraná, considerando os 37 anos da série histórica analisada.

No tocante as áreas em que os totais pluviométricos anuais tendem a diminuir sobressaem as mesorregiões Centro-Oriental, Norte Central (Maringá), Sudoeste e Sudeste, mas com índices pouco expressivos que variam de $-0,05$ a -0,23 para o teste da Correlação Linear e de -0,04 a -0,18 para o teste de Mann-Kendall. Com os resultados do teste da tendência Linear (OLS) identificase que ocorre uma diminuição de 55 a 300mm nas chuvas anuais; considerando uma redução $-1,50$ a $-10,22 \mathrm{~mm}$ para cada ano da série histórica.

Em síntese, as tendências mais significativas observadas no estado são aquelas que indicam aumento nos totais pluviométricos anuais. Aspecto que merece atenção dos órgãos de planejamento, pois áreas que já são úmidas apresentam um aporte em suas precipitações anuais que poderão desencadear diversos impactos, principalmente sobre as bacias hidrográficas e, mais especificamente, sobre as áreas urbanas. Enquanto que as áreas mapeadas com tendências à redução, mesmo não sendo significativas estatisticamente, merecem uma análise mais detalhada para a identificação de períodos mais específicos em que se manifestam tais reduções; pois podem afetar sobremaneira a economia do estado, baseada nas atividades agrícolas.

\section{REFERÊNCIAS}

BACK, J. Á. Aplicação de análise estatística para identificação de tendências climáticas. Pesquisa Agropecuária Brasileira. Brasília, v.36, n.5, maio/2001. p. 717-726

BARROS, V. Introduction. In: BARROS, V.; CLARKE, R.; SILVA DIAS, P. Climate change in the La Plata basin. Inter-American Institute for Global Change (IAI), 2010. p. 8-15

CARMELLO, V., DUBREUIL, V., SANT'ANNA NETO, J.L. Analyse géostatistique de la distribution spatiale des précipitations sur le versant sud du bassin du fleuve Paranapanema, dans le sud du Brésil. Actes du XXVIIIe Colloque de I'Association Internationale de Climatologie, Liège, juillet 2015. p.439-444

CHAKRABORTY, S.; PANDEY, R. P.; CHAUBE, U. C.; MISHRA, S. K. Trend and variability analysis of rainfall series at Seonath River Basin, Chhattisgarh (India). International journal of Applied Sciences and Engineering Research, v. 2, Issue 4, 2013. p. 425-434. Doi: 10.6088/ijaser.020400005 
DEBORTOLI, N., DUBREUIL, V., HEINKE, C., RODRIGUEZ FILHO, S. Tendances et ruptures des séries pluviométriques dans la région méridionale de l'Amazonie brésilienne. Actes du 25e Colloque de I'AIC, Grenoble, sept.2012. pp.201-206

DUBREUIL, V.; MEROT, P.; DELAHAYE, D.; DESNOS, P. Changement climatique dans I'Ouest. Rennes, Presses Universitaires de Rennes, 2012, 464 p.

DUBREUIL, V.; FANTE, K. P.; PLANCHON, O.; SANT'ANNA NETO, J. L. Les types de climats annuels au Brésil: une application de la classification de Köppen de 1961 à 2015. EchoGéo, 41, 2017. DOI: 10.4000/echogeo.15017

ELY, D., DUBREUIL, V. Evolutions des températures et des précipitations dans le versant sud du bassin de la rivière Paranapanema, Paraná, Brésil. Actes du 27e colloque de I'AIC, Dijon, 2014. pp.555-560

FERREIRA, M., C. Iniciação à análise geoespacial: teoria, técnicas e exemplos para geoprocessamento. São Paulo: Editora UNESP, 2014.

FERREIRA, D. H. L.; PENEREIRO, J. C.; FONTOLAN, M. R. Análises estatísticas de tendências das séries hidro-climáticas e de ações antrópicas ao longo das subbacias do rio Tietê. Holos, ano 31, v.2, 2015. DOI: 10.15628/holos.2015.1455

HAYLOCK, M. R.; PETERSON, T.; ABREU DE SOUSA JR, et al. (2006) Trends in total and extreme South American temperature 1960-2000 and links with sea surface temperature. Journal of Climate, 9:1490-1512.

IPCC. Climate Change 2014: Synthesis Report. Contribution of Working Groups I, II and III to the Fifth Assessment Report of the Intergovernmental Panel on Climate Change [Core Writing Team, R.K. Pachauri and L.A. Meyer (eds.)]. IPCC, Geneva, Switzerland, 2014. 151 pp.

NASCIMENTO JUNIOR, L., RODRIGUES, M.S., DUBREUIL, V., SANTA'ANNA NETO, J.L. Rupturas e tendências em séries históricas no Paraná. Anais do XV Simposio Brasileiro de Geografia Física Aplicada. Vitória, 2013. pp. 298-307; disponível

em

http://www.xvsbgfa2013.com.br/anais/trabalhos/Eixo02.pdf\#page $=298$

MENDONÇA, F. A. O Clima e o Planejamento Urbano de Cidades de Porte Médio e Pequeno. Proposição Metodológica para Estudo e sua Aplicação à Cidade de Londrina/Pr. 1994. Tese (Doutorado) - Faculdade de Filosofia Letras e Ciências Humanas, Universidade de São Paulo. São Paulo.

Aquecimento global e suas manifestações regionais e locais: alguns indicadores da região Sul do Brasil. Revista Brasileira de Climatologia. Presidente Prudente: ABClima, v. 2, n. 2, 2006. p. 71 - 86

.(Org.) Os Climas do Sul: em tempos de mudanças climáticas globais. Jundiaí, Paco Editorial, 2014, 276p.

MINUZZI, R. B.; CARAMORI, P. H. Variabilidade climática sazonal e anual da chuva e veranicos no Estado do Paraná. Viçosa: Revista Ceres, v. 58, n.5, set/out, 2011. p. 593-602

MOREIRA, J. G. do V.; NAGHETTINI, M. Detecção de tendências monotônicas temporais e relação com erros dos tipos I e II: estudo de caso em séries de precipitações diárias máximas anuais do estado do Acre. Revista Brasileira de Meteorologia, v. 31, n. 4, 2016. p. 394-402, DOI: http://dx.doi.org/10.1590/0102-778631231420140155 (2016 
ROGERSON, P. A. Métodos estatísticos para geografia: um guia para o estudante. Porto Alegre: Bookman, 2012.

SALVIANO, M. F.; GROPPO, J. D.; PELEGRINO, G. Q. Análise detTendências em dados de precipitação e temperatura no Brasil. Revista Brasileira de Meteorologia, v. 31, n. 1, 2016. p. 64-73 DOI: http://dx.doi.org/10.1590/01027786201500032016

SILVA, M. E. S.; GUETTER, A. K. Mudanças climáticas regionais observadas no estado do Paraná. Terra Livre. São Paulo: Associação dos Geógrafos Brasileiros (AGB), Ano19, v. I, n.20, jan/jul 2003. p. 111 - 126

TOZATO, H., DUBREUIL, V., DE MELLO-THÉRY, N.A. Tendências e rupturas climato-hidrológicas no sitio ramsar PARNA Pantanal (MT, Brasil). Revista Brasileira de Climatologia. 2013, vol.9. pp.164-184 